\title{
Tratamiento del síndrome genitourinario de la menopausia mediante láser fraccionado CO2: una opción terapéutica emergente
}

\author{
Juan José Escribano $T^{1,2^{*}}$, Gloria Rodea G. ${ }^{1,3^{*}}$, Ángel Martín J. ${ }^{4,5^{*}}$, Ignacio Cristobal \\ G. ${ }^{*}$, Pedro Jesús González . $^{7^{*}}$, Joan Salinas P. ${ }^{8,9^{\star}}$, Rafael Sánchez-Borrego. ${ }^{\star *}$ \\ ${ }^{1}$ Unidad Ginecología Estética y Funcional. Instituto Médico Láser, Madrid. ${ }^{2}$ Servicio de Ginecología y Obstetricia, \\ Hospital Universitario Severo Ochoa, Leganés, Madrid. ${ }^{3}$ Unidad de Ginecología, Centro Médico Gran Vía, Madrid. \\ ${ }^{4}$ Unidad Ginecología Estética y Funcional, Clínica Juaneda, Palma de Mallorca, 5 Servicio de Ginecología y Obstetricia, \\ Hospital Son Llatzer, Palma de Mallorca. ${ }^{6}$ Hospital Universitario Sanitas La Zarzuela, Madrid, ${ }^{7}$ Hospital Universitario \\ Miguel Servet, Zaragoza. ${ }^{8}$ Hospital Universitario Sant Joan de Reus, Tarragona. ${ }^{9}$ Clínica Biogest, Reus, Tarragona. \\ 10 Instituto Ginecología y Obstetricia DIATROS, Barcelona. España.
}

* Grupo de Ginecología Estética y Funcional. Asociación Española Estudio Menopausia (AEEM)

\section{RESUMEN}

Estudios recientes han demostrado que el láser fraccionado es una opción terapéutica no hormonal efectiva, sencilla, bien tolerada y sin efectos adversos, para el tratamiento del Síndrome Genitourinario de la Menopausia. La reacción térmica originada produce la restauración del epitelio vaginal, un proceso de neocolagénesis y una mayor vascularización que favorece la llegada de nutrientes, reestableciéndose la estructura de la mucosa, su grosor y trofismo funcional, mejorando por tanto la sintomatología. No obstante, se precisan estudios a largo plazo, controlados, frente a estrógenos locales y otros tratamientos no hormonales para validar la duración de los efectos y la seguridad de las sucesivas aplicaciones. El objetivo de este trabajo es revisar la evidencia relacionada con esta terapia emergente.

\section{PALABRAS CLAVE: Láser, atrofia vaginal, síndrome genitourinario, menopausia}

\section{SUMMARY}

Recent reported studies have demonstrated that non-invasive fractional laser is a valid, safe, effective and well tolerated therapeutic option, without adverse events to treat the Menopause Genitourinary Syndrome. The heat shock effect induces the increase of vaginal epithelium thickness, the new glycogen content and the vascular changes, improving the vaginal epithelium structure, functionality and menopausal symptoms. Nevertheless, duration of treatment effects and safety of repeated session are not clear enough. Further controlled long-term follow-up research on laser versus local estrogens and other non-hormonal therapies are needed. The objective of this paper is to review the scientific evidence related to this emergent treatment.

KEY WORDS: Laser, vaginal atrophy, genitourinary syndrome, menopause 


\section{INTRODUCCIÓN}

La carga genética determina, junto con la influencia de los factores ambientales, el proceso de envejecimiento en la mujer. La depleción de los niveles de estrógenos (E), que ocurre después de la menopausia origina una serie de modificaciones en numerosas zonas del cuerpo femenino, incluyendo el suelo pélvico y el área genital, debido a la presencia de receptores de estrógenos en el tracto urogenital inferior y genitales externos (trígono, uretra, vagina, vulva, vestíbulo y labios). La menopausia causará en algún momento, síntomas genitourinarios importantes que afectarán la calidad de vida (CV) de la mujer $(1,2)$.

Hay 64 millones de mujeres postmenopáusiacas en Estados Unidos y aproximadamente $32 \mathrm{mi}-$ llones de ellas pueden sufrir signos y síntomas de atrofia vulvovaginal (AVV), como sequedad vaginal, sensación de escozor, picor, irritación o quemazón y dispareunia (Tabla I). La AVV severa puede interferir en otros aspectos de la CV de las mujeres tales como la práctica de ejercicio, el uso de determinadas prendas de vestir y en aspectos generales relacionados con el normal funcionamiento del suelo pélvico (3-6) (Tabla II).

La AVV se definía como la involución crónica y progresiva de los tejidos de la vulva y vagina consecuencia de la pérdida de $E$ durante la menopausia, originándose un estrechamiento y acortamiento vaginal y un cierto grado de estenosis del introito (7-11). Pero este concepto clásico de AVV se modificó recientemente por la International Society for the Study of Women's Sexual Health (ISSWSH) y la North American Menopause Society (NAMS), por una nueva y más amplia definición, el síndrome genitourinario de la menopausia (SGM). La intención

Tabla I

SÍNTOMAS DE AVV REFERIDOS POR LAS PARTICIPANTES DEL ESTUDIO REVIVE (3)

\begin{tabular}{lc}
\hline Síntoma & $\begin{array}{c}\text { Participantes en estudio } \\
\text { REVIVE que refieren } \\
\text { síntomas de AVV (\%) }\end{array}$ \\
\hline Sequedad vaginal & 55 \\
Dispareunia & 44 \\
Irritación vaginal & 37 \\
Sensibilidad vaginal & 17 \\
Sinusorragia & 8 \\
Dolor durante el ejercicio & 2 \\
\hline
\end{tabular}

REVIVE: REal Women's Vlews of Treatment Options for Menopausal Vaginal ChangEs. AVV: atrofia vulvar y vaginal.

\section{Tabla II}

PORCENTAJE DE MUJERES QUE REFIEREN PROBLEMAS/INTERFERENCIAS, DEBIDO A LOS SÍNTOMAS VULVARES Y VAGINALES (3)

\begin{tabular}{lc}
\hline Actividad & $\begin{array}{c}\text { Mujeres que } \\
\text { refieren } \\
\text { interferencias } \\
(\%)\end{array}$ \\
\hline Disfrutar con las relaciones sexuales & 63 \\
Sensación de espontaneidad sexual & 55 \\
Habilidad para intimar & 54 \\
Capacidad de relacionarse & 45 \\
Conciliar el sueño & 29 \\
Disfrutar de la vida en general & 27 \\
Temperamento & 26 \\
Búsqueda de una nueva relación íntima & 13 \\
Viajar & 13 \\
Actividades deportivas & 12 \\
Actividades cotidianas & 11 \\
Participación en actividades sociales & 10 \\
Capacidad de trabajo & 7 \\
\hline
\end{tabular}

es integrar la enorme variedad de signos y síntomas asociados a los progresivos cambios vulvovaginales (labios mayores y menores, clítoris, vulva, vestíbulo, introito y vagina) y que abarca también al tracto urinario inferior (vejiga, uretra), todos ellos relacionados con la deficiencia estrogénica y de otros esteroides sexuales (12-14). La expectativa de vida en la mujer para el año 2050 es de 81 años y la edad media de la menopausia es 51 años, por tanto, muchas de ellas vivirán un $40 \%$ de sus vidas afectadas por el SGM $(14,15)$.

\section{SÍNDROME GENITOURINARIO DE LA MENO- PAUSIA}

Los síntomas y signos del SGM afectan de manera progresiva en la menopausia desde su aparición, a diferencia de los síntomas vasomotores que mejoran o desaparecen con el paso del tiempo. Las molestias más frecuentes en pacientes con SGM son la sequedad vaginal, prurito, ardor disuria y dispareunia, que se asocian con frecuencia a problemas cutáneos en los genitales externos. Como consecuencia, la calidad de vida se ve afectada en un $30-50 \%$ en este grupo poblacional. Además, las infecciones vaginales de repetición ocurren por la alteración de la microbiota vaginal (disminución de 
lactobacilos y aumento de bacterias patógenas), originada por la pérdida del contenido de glucógeno de la mucosa vaginal derivada del cese de la función ovárica (16).

El diagnóstico del SGM se basa en la historia clínica, orientada hacia la presencia de síntomas genitourinarios, incluida la incontinencia urinaria de urgencia y un aumento de infecciones de orina, y en la exploración clínica, que revelará una disminución de los pliegues vaginales o adelgazamiento de la mucosa y afectación de la piel de la vulva (13).

La pérdida de $E$ y la edad originan cambios anatómicos, fisiológicos y clínicos que ocurren en el SGM. Hasta la menopausia, la vagina presenta unas rugosidades características. La mucosa vaginal consta de epitelio y lámina propia sin plano submucoso, como ocurre en la piel. El epitelio está formado por varias capas de células ricas en glucógeno y los $\mathrm{E}$ favorecen su correcto desarrollo, constituyendo un epitelio escamoso poliestratificado (17-20 capas) no queratinizado. Las células epiteliales se van renovando y liberan glucógeno que es hidrolizado a glucosa, transformada en ácido láctico mediante la acción de los lactobacilos de la flora.

La lámina propia está integrada por tejido conectivo rico en colágeno y fibras elásticas, no contiene glándulas secretoras propias, pero sí abundantes fibroblastos y fibrocitos, con numerosos vasos, y es la responsable de la nutrición, soporte y arquitectura de las paredes vaginales. Debido a esta estructura, la vagina presenta esa consistencia elástica y flexible típica (12,14-18). La progresiva reducción de los $\mathrm{E}$ circulantes que ocurre durante la menopausia, induce cambios matabólicos y tisulares, más importantes en el área geintourinaria, debido a su mayor sensibilidad a los cambios en los niveles de las hormonas sexuales $(7,13,16-20)$. Por ello, el epitelio vaginal se adelgaza y existe una menor cantidad de glucógeno en las células superficiales con las consecuencias en la flora vaginal y el aumento del $\mathrm{pH}$. Los cambios en la microbiota originan un crecimiento de bacterias patógenas que pueden originar infecciones recurrentes. La superficie vaginal aparece más friable y con mayor sequedad, pudiendo sangrar después de mínimos traumas, perdiendo su característico aspecto rugoso, y en la zona vulvar, el clítoris se atrofia, junto con los labios menores y mayores, objetivándose en ocasiones una carúncula uretral, consecuencia de la prominencia del meato urinario $(14,21)$.

Además, se produce una alteración en la proporción de fibras de colágeno tipo I y tipo III con una pérdida en su disposición trabecular, una disminución del número de fibras elásticas, una menor vascularización y un adelgazamiento del epitelio vaginal que puede originar en las capas más su- perficiales zonas de queratinización. Como consecuencia, el canal vaginal se estrecha y se acorta, perdiendo su elasticidad y los típicos pliegues vaginales. El epitelio se hace más pálido, fino y con mayor presencia de petequias $(1,7,22)$. Además, la secreción originada mediante transudado de los tejidos vecinos disminuye, afectando a la lubricación que se reduce enormemente durante la relación sexual $(7,11,12,14,22-25)$.

Todas estas modificaciones clínicas se asocian con la pérdida de actividad sexual y/o con disfunciones sexuales en la mujer $(4,26,27)$. Las pacientes con SGM se quejan de quemazón, prurito, sequedad, irritación, escozor, dispareunia y disuria (28-31), y los tejidos debilitados son más propensos a desarrollar traumas, infecciones y sangrados (7). Estudios sobre la prevalencia de los síntomas del SGM indican que aproximadamente el $50 \%$ de las mujeres postmenopáusicas refieren como mínimo uno de los síntomas descritos $(8,32)$. A pesar del impacto negativo en la $\mathrm{CV}$, en el ámbito sexual $y$ en las relaciones interpersonales durante esta etapa, estas cuestiones son todavía un tabú en las consultas de ginecología y somos los profesionales de la salud los que debemos mantener una actitud proactiva al respecto, procurando una adecuada educación sanitaria, un diagnóstico temprano y un consenso sobre las opciones terapéuticas, para establecer la más adecuada en función de los síntomas $(8,21,32-35)$, porque la clínica puede empeorar con el paso de los años, agravando los indicadores de CV (36).

En el estudio VIVA (Vaginal Health: Insight, Views and Attitudes), casi la mitad de las mujeres postmenopáusicas presentan síntomas de intensidad variable, desde leves y moderados hasta severos e intolerables, pero solo un $25 \%$ de ellas solicitaron asistencia médica, debido a la sensación de vergüenza y a la creencia de que los síntomas eran normal consecuencia del envejecimiento $(34,35)$. En el estudio Women's Voices in Menopause, se evidenció el impacto del SGM en la CV en el 52\% de las mujeres, incluyendo indicadores sobre salud sexual, autoestima, relaciones de pareja y relaciones sociales (33). Estos estudios corroboran que debido al progresivo envejecimiento de la población, las mujeres podrán verse afectadas por el SGM durante más de un tercio de su vida (4,33-38).

Las mujeres postmenopáusicas que han padecido un cáncer de mama presentan síntomas del SGM en un $70 \%$ de los casos, comparadas con el $50 \%$ de las no afectas, y sobre todo las pacientes más mayores que han recibido tratamientos farmacológico (quimioterapia y/o hormonoterapia). Los inhibidores de la aromatasa se relacionan especialmente con la afectación vaginal y con la pérdida de calidad de vida en ese grupo de mujeres $(39,40)$. 


\section{OPCIONES TERAPÉUTICAS DEL SGM}

Hoy en día tenemos diferentes opciones terapéuticas para aliviar los síntomas del SGM. Cuando la terapia hormonal sustitutiva (THS) se utiliza solo para el tratamiento de la atrofia vaginal, los E locales representan la alternativa de primera línea (4350). Sin embargo, la aceptación, la adhesión y la preferencia por este tratamiento de las mujeres es relativamente pobre (14,38-43). Otras modalidades terapéuticas paliativas de los síntomas, tales como hidratante y/o lubricantes en forma de crema o gel, se han propuesto con frecuencia y aunque mejoran los síntomas inicialmente, no presentan efectos terapéuticos a largo plazo ni mejoran los indicadores de maduración vaginal $(1,4,7,13,14,42,44-52)$. Así, las últimas guías clínicas indican diferentes opciones en función de la severidad de los síntomas, de su eficacia y seguridad y de las preferencias de las pacientes $(4,7,14,26,27,47)$.

Los lubricantes son utilizados principalmente para aliviar la sequedad vaginal durante la relación sexual pero los estudios no les avalan como solución a largo plazo, mientras que el uso continuo de los hidratantes vaginales puede tener un efecto beneficioso duradero especialmente si la paciente tiene relaciones sexuales con regularidad. Un reciente modulador selectivo de los receptores de E, ospemifeno, ha sido aprobado como tratamiento de la dispareunia en mujeres postmenopáusicas con SGM, demostrándose efectivo y seguro en la pauta diaria de $60 \mathrm{mg}$ en más del $93 \%$ de los casos durante más de 1 año $(13,14,17,47-49)$.

La THS sistémica debe considerarse para los síntomas climatéricos, especialmente sofocos, en ausencia de contraindicaciones; sin embargo, esta terapia puede asociarse con más efectos secundarios que la terapia local, por lo que ésta resulta de elección para mejorar la sintomatología y revertir los cambios histológicos que sufre la vagina, en los casos en que la AVV represente el único cuadro clínico de la mujer $(7,14,43,48,50)$. Aunque el tratamiento local con $E$ es efectivo y seguro a dosis muy bajas $(8,10,14,23,38,43)$, el porcentaje de adhesión al mismo es bastante variable (52-74\%) (51), principalmente en lo relacionado a la seguridad a largo plazo y a la ausencia de alivio uniforme de los síntomas $(52,53)$. Faltan datos de seguridad a largo plazo, más de 1 año, sobre el uso local de $E$ y aunque no parece incrementar el riesgo trombo embolismo venoso, no existe evidencia disponible en mujeres de alto riesgo $(7,17-19,27,46,47,49,52-$ 54). Además, las mujeres con cáncer ginecológico estrógeno-dependiente (fundamentalmente mama), presentan un alto riego de AVV asociado a problemas importantes relacionados con la esfera sexual $(39,40,54)$, y requieren una especial consideración y consenso individualizado antes de prescribir cualquier tipo de tratamiento con $\mathrm{E}$ local $(7,14,17,38,55-58)$. Por otra parte, la mayor desventaja que presenta esta pauta local, es la recurrencia de los síntomas una vez suspendido el tratamiento, resultando únicamente efectivo en las capas más superficiales del epitelio vaginal (17).

\section{TRATAMIENTO DEL SGM CON LÁSER FRAC- CIONADO}

En el ámbito de la medicina actual y desde hace años, la terapia "antiaging" ofrece una serie de alternativas para eliminar los daños o efectos deletéreos que el envejecimiento fisiológico produce en el organismo, restaurando, regenerando o restableciendo el aspecto y funcionalidad normal de nuestro cuerpo. En este campo se aplica la tecnología láser, para que sea el propio organismo quién active los mecanismos biológicos propios de reparación tisular. Es en el proceso del envejecimiento cutáneo, en el que la utilización del láser fraccionado microablativo de dióxido de carbono (CO2), ha generado una enorme expectativa y aceptación, aplicándose fundamentalmente en dermatología y cirugía plástica, como una opción terapéutica regenerativa no invasiva, dada su eficacia y precisión.

El láser CO2 tiene además muchas aplicaciones en otras especialidades médicas como la podología, otorrinolaringología, ginecología, traumatología, neurología, cirugía torácica, cirugía maxilofacial y cirugía uroginecológica. Permite la incisión, excisión, vaporización y coagulación tisular, por lo que desde sus inicios se ha utilizado para el tratamiento de lesiones cutáneas del cuerpo, cuello y cara (queratosis seborréicas, xantelasmas, etc.), lesiones de la mucosa oral, condilomas acuminados, enfermedades de las uñas, etc. Además, tiene una función ablativa indicada para el antienvejecimiento cutáneo (resurfacing-reshaping) que gana más adeptos día a día (1), debido a su seguridad y efectos de remodelado tisular $(1,7,14,17,38,59$ 62). Por ello, y basado en los resultados obtenidos sobre la piel, el uso del laser fraccionado de $\mathrm{CO} 2$, específicamente diseñado para la mucosa vaginal con unos parámetros predeterminados, ha sido publicado como tratamiento satisfactorio para los síntomas del SGM, sin efectos secundarios, lesiones posteriores y muy bien tolerado por las pacientes $(1,7,14,17,33)$. Desde que la US Food and Drug Administration (FDA) aprobara en 2014 esta tecnología para uso vaginal, otros sistemas láser que utilizan medios activos diferentes al $\mathrm{CO} 2$ se han posicionado también en el mercado (63). Así, diferentes publicaciones han comunicado recientemente la utilidad del láser Erbio:YAG (VEL) como tratamiento del SGM $(38,64-69)$.

El mecanismo de acción del láser $\mathrm{CO} 2$ fraccionado microablativo permite su aplicación en casos de piel atrófica, evidenciándose unas propiedades regenerativas por la contracción tisular generada 
(70-74), con cambios histológicos y moleculares importantes en los componentes del tejido conectivo, que son igualmente observados cuando se aplica a la mucosa vaginal mediante un tipo de pulso específico para esta zona (75). Los efectos conseguidos ocurren gracias a la microablación que estimula el remodelado tisular. Este proceso es debido a la reacción térmica generada que ocasiona la activación del las proteínas "heat schock proteins" (HSP), 43, 47 y $70(74,76)$. Estas proteínas desencadenan un incremento local de varias citokinas, destacando: el factor de crecimiento A (que estimula las proteínas de la matriz extracelular, como el colágeno); el factor de crecimiento de los fibroblastos (que estimula la síntesis de colágeno); factores angiogénicos (con aumento de migración y proliferación de células endoteliales); el factor epitelial de crecimiento (que estimula la reepitelización); el factor de crecimiento plaquetario (estimula los fibroblastos para producir nuevo colágeno y otros componentes de la matriz extracelular); y el factor de crecimiento endotelial vascular (que regula la vasculogénesis y angiogénesis). El conjunto de estas interacciones derivadas de la energía térmica aplicada sobre el tejido es la activación de la célula clave o fibroblasto, que origina la producción de nuevas fibras de colágeno, fibras elásticas y otros componentes de la sustancia extracelular (proteoglicanos, glicosaminglicanos, ácido hialurónico y otras moléculas), y la neovascularización, con efectos nutritivos específicos sobre el tejido epitelial (77).

Basados en los datos publicados hasta la fecha, las primeras experiencias con estudios pilotos a corto y medio plazo, muestran que el tratamiento mediante laser fraccionado de $\mathrm{CO} 2$, con una adecuada selección de pacientes (Tablas III y IV), constituye una nueva opción terapéutica no hormonal para las mujeres postmenopáusicas con SGM, factible, eficaz y muy bien tolerada $(7,17,38)$.

\section{Tabla III CRITERIOS DE INCLUSIÓN (TRATAMIENTO LÁSER) (7)}

\footnotetext{
- Menopausia establecida (incluyendo menopausia precoz): mínimo 12 meses desde la última menstruación o de una ooforectomía bilateral
}

- Síntomas de AVV: sequedad vaginal y/o picor, quemazón y/o dispareunia (78)

- Sin respuesta o respuesta insatisfactoria al tratamiento previo con estrógenos u otras terapias locales

AVV: atrofia vulvar y vaginal.

\section{Tabla IV \\ CRITERIOS DE EXCLUSIÓN (TRATAMIENTO LÁSER) (7)}

\footnotetext{
- Infección urinaria aguda o de repetición
}

- Infección genital activa

- Prolapso genital (POP): estadio>II-Half Way System for quantification POP (79)

- Utilización de Terapia Hormonal Sustitutiva sistémica o local hasta 6 meses previos a la inclusión en el estudio

- Uso de lubricantes, hidratantes o cualquier otro tratamiento local dentro de los 30 días previos a la inclusión en el estudio

- Cirugía previa del suelo pélvico

- Enfermedad grave y/o crónica que afecte al cumplimiento del estudio

- Enfermedades psiquiátricas

- Uso de drogas psicotrópicas 


\section{SISTEMA LÁSER CO2 FRACCIONADO}

El sistema de láser CO2 fraccionado (SmartXide 2V2LR,Deka-,Florence, Italy) está equipado con un escáner V2LR (VulvoVaginal Laser Reshaping), y diferentes terminales específicos para vagina. Este tratamiento se basa en la interacción entre un tipo de pulso de emisión del $\mathrm{CO} 2$ y la mucosa vaginal. El haz láser es emitido de forma fraccionada y focalizada en diferentes y pequeños puntos, denominados DOT, separados entre sí por islotes de tejido sano. El láser penetra en los tejidos y actúa sólo cuando la profundidad de penetración establecida es alcanzada. Mediante un software es posible seleccionar el modo específico de pulso (D-Pulse), la profundidad (SmartStak: 1-3) y la cantidad (potencia, tiempo de exposición "dwell time" y distancia entre los puntos), de calor que se puede transmitir al tejido. La función SmartStack permite un cuidadoso control de la profundidad de vaporización inicial y de la acción térmica. Pulsos sucesivos son emitidos en la misma zona del tejido vaginal en función de la variable 1-3, facilitando que la mucosa se refrigere entre dos pulsos sucesivos y minimizándose así el daño térmico. Cada pulso está compuesto por un pico de liberación de alta energía que produce una rápida ablación del tejido epitelial de la mucosa atrófica vaginal, seguido de una emisión de energía más larga y duradera que permite que el efecto del láser $\mathrm{CO} 2$ penetre más profundamente en la mucosa. Los pulsos están distribuidos por todas las paredes vaginales y espaciados de forma que cubran la totalidad del área a tratar (7). Un terminal específico se introduce con facilidad en la vagina y permite la transmisión del pulso, dispersando la energía en $360^{\circ}$. El láser es proyectado hacia un espejo piramidal orientado $45^{\circ}$ que se coloca en el extremo distal del terminal para reflejarse de esta manera hacia todas las paredes de la vagina al mismo tiempo, pero no hacia el cérvix uterino. Para completar el tratamiento de todo el canal vaginal es necesario emitir sucesivos spots con el láser mientras extraemos el terminal progresivamente desde el fondo vaginal, guiados por las marcas que aparecen en aquel y que nos indican cada cuántos milímetros debemos emitir los pulsos (manteniendo la misma distancia). Existen otros terminales que permiten la aplicación sucesiva en las caras anterior, lateral, posterior y lateral opuesta de la vagina mediante su rotación (utilizando un dispositivo especial) en sentido horario (7).

\section{PROTOCOLO DE APLICACIÓN}

Los parámetros prefijados para el tratamiento fraccionado con láser $\mathrm{CO} 2$ se han seleccionado en base a los datos obtenidos "ex vivo", en muestras de pared vaginal, que ponen de manifiesto la remodelación tisular sin evidencia de lesión en la zona periférica $(1,7)$. Cuando es necesario, la potencia DOT se reduce para el tratamiento del introito vaginal, dada la mayor sensibilidad de la zona.

Las pacientes no suelen presentar molestias durante la aplicación al utilizar los valores adecuados para cada tipo de tratamiento (17), y en las 3 sesiones que se realizan el protocolo de actuación en cada una de ellas consta de dos fases:

1) Mediante la colocación del espéculo se observa la vagina para determinar el VHIS (Vaginal Health Score Index) o Índice de Salud vaginal, que se realiza en cada sesión (80).

2) Se introduce con cuidado el terminal hasta el fondo de la vagina antes de comenzar con el procedimiento.

El ciclo de tratamiento incluye tres sesiones y los controles habituales son en la primera sesión (visita basal), a las 4 semanas (visita 1) y a las 8 semanas de la primera aplicación (visita 2) o fin de tratamiento. En la literatura se han descrito publicaciones con controles a las 4 semanas de la última sesión y en periodos más largos para evaluar la efectividad a largo plazo y la aparición de efectos secundarios, todos con excelentes resultados $(7,17,38)$. El procedimiento se realiza en consulta externa y no requiere preparación específica (lubricante, analgesia, anestesia, etc.). Se recomienda a las pacientes evitar las relaciones sexuales durante 2-3 días posteriores a la sesión, debido a la leve reacción inflamatoria que puede ocurrir en las primeras 48 horas.

Como se ha comentado anteriormente, cada paciente es evaluada en cada sesión utilizando el VHIS, integrado por la medida de 5 parámetros: elasticidad, fluidez y consistencia de la secreción, $\mathrm{pH}$ vaginal, integridad del epitelio y grado de hidratación. Cada parámetro se valora desde 1 (peor valoración) hasta 5 (mejor valoración), considerando la vagina atrófica si la puntuación total es inferior a $15(80,81)$.

El grado de elasticidad se evalúa principalmente mediante la colocación del espéculo en la vagina y en función de la severidad de la atrofia es posible objetivar menor capacidad de distensión del canal vaginal y molestia o dolor en esta fase. Además, la aplicación de una torunda de algodón presionando en las paredes vaginales permite aumentar la valoración de la capacidad elástica de la vagina. La integridad de la mucosa vaginal también puede ser valorada durante la introducción del espéculo, observando la tendencia al sangrado de las paredes durante ese momento. El pH vaginal se mide utilizando un test colorimétrico habitual y para evaluar la consistencia de la secreción se utiliza una torunda de algodón aplicada por las paredes vaginales. Por último, el grado de hidratación se comprueba mediante visión colposcópica de la superficie vaginal. 
Por otra parte, la intensidad de los síntomas del SGM (quemazón, prurito, sequedad, dispareunia y disuria) se mide utilizando una escala analógica visual de $10 \mathrm{~cm}$ de longitud (VAS), mediante la cual, la paciente nos informa sobre su clínica, conociendo que el extremo izquierdo de la escala (valor 1) indica la ausencia de síntomas y el extremo derecho (valor 10) indica el mayor grado de severidad (el peor grado soportable). Esta escala visual se evalúa antes de cada aplicación del láser.

Como complemento a dichos sistemas de evaluación del tratamiento, con el ánimo de recabar la mayor información posible y después de cada sesión, el profesional valora el grado de dificultad encontrada durante el procedimiento mediante la escala de 5 puntos de Likert (muy fácil, fácil, normal, difícil y muy dificultoso), y la paciente nos informa del grado de molestia y/o dolor percibido en el momento de la inserción y durante las maniobras de realización de la técnica, utilizando la VAS, en la que el valor 1 indica ausencia de dolor y el valor 10 refleja el peor dolor posible (7).

En el seguimiento posterior a medio-largo plazo algunos grupos preguntan a las pacientes sobre el grado de satisfacción global con el tratamiento mediante la siguiente cuestión: Teniendo en cuenta las modificaciones percibidas en sus síntomas, su grado de bienestar y calidad de vida, y considerando los efectos adversos experimentados, en caso de que hayan ocurrido, ¿Cómo definiría el grado de satisfacción con la técnica láser? (7). Las respuestas se recogen mediante la escala 5-Likert (muy satisfecha, satisfecha, indiferente, insatisfactoria, muy insatisfecha). La satisfacción con el tratamiento se considera con las respuestas "muy satisfecha" o "satisfecha". Debido al análisis estadístico que se realiza habitualmente (intención de tratar), todas aquellas mujeres que son incapaces de tolerar la inserción del terminal debido a la severidad de los síntomas del SGM y/o a la presencia de estenosis vaginal, se consideran "muy insatisfechas" (7) .

Para la evaluación de los posibles efectos adversos ocurridos durante el tratamiento, inmediatamente después de cada sesión y hasta el final del proceso de análisis de resultados, se consideran como tales cualquier alteración, sensación de disconfort o lesión a nivel local o general, acontecidos en relación con la aplicación del láser fraccionado.

\section{DISCUSIÓN}

El término "láser" es un acrónimo de "Light Amplification by Stimulated Emission of Radiation". El haz emitido es una radiación electromagnética, similar a la luz natural, pero generada mediante emisiones estimuladas. La luz generada es monocromática y coherente, que significa que es emitida en una misma longitud de onda, temporal y espacialmente en fase. Cada tipo de láser se define por su longitud de onda, que determina el color de la luz generada y también por su medio activo dónde la energía es estimulada. Este medio puede ser un gas, un cristal o un líquido y se encuentra almacenado en una cavidad óptica de resonancia, en cuyos extremos se encuentran unos espejos a través de los cuales entra la luz a la caja de resonancia con el medio apropiado, es estimulada y sale por el espejo distal convertida en energía láser. En la actualidad existen más de 40 tipos de láser que han sido desarrollados para un amplio abanico de posibilidades, tanto en la industria como en el ámbito de la medicina. Los láser con medio activo gaseoso y sólido son utilizados frecuentemente para intervenciones quirúrgicas, fundamentalmente los de $\mathrm{CO} 2$, Argón, Neodimio:YAG y el KTP:YAG (82). El láser de CO2 fue desarrollado en 1964 por Kuman Patel (83) y fue aplicado por primera vez en ginecología en 1973, para el tratamiento de las erosiones cervicales, y posteriormente en 1977 para la neoplasia cervical intraepitelial y para la microcirugía de las trompas de Falopio en 1983. Sus ventajas para el tratamiento de los casos de CIN, VIN, VAIN y enfermedad condilomatosa han sido ampliamente documentadas durante los últimos años (82,84-86). El láser $\mathrm{CO} 2$ es considerado actualmente como el más versátil y seguro y con los últimos avances en los sistemas de transmisión del haz de luz (fibras flexibles fotónicas), puede ser utilizado con mayor facilidad en la cirugía endoscópica, ofreciendo nuevas alternativas terapéuticas para el tratamiento de la endometriosis y el síndrome adherencial (82).

La interacción de la energía láser con los tejidos puede originar diversos efectos, incluyendo el efecto fototérmico, fotoquímico y fotoacústico. Cuando el haz impacta sobre la superficie tisular, los fotones pueden ser absorbidos, reflejados o dispersados. Solo la luz absorbida es capaz de convertirse en calor y es este efecto fototérmico relacionado con la absorción, el más aplicado por la energía láser en ginecología (82). Debido a que la distribución de la energía en la zona tratada no es uniforme, el impacto del láser en los tejidos origina un cráter con tres zonas bien definidas de lesión: Vaporización central en forma de V, necrosis subyacente con sellado de vasos $<1 \mathrm{~mm}$ y una zona más inferior de lesión térmica reversible (82).

Hasta la fecha, el uso del láser $\mathrm{CO} 2$ ha sido ampliamente aceptado en medicina, especialmente en dermatología y cirugía plástica $(61,62,72,76)$. Este láser consiste en rayos infrarrojos de $\mathrm{CO} 2$ que generan una reacción térmica exagerada (HRS: Heat Shock Response) y vaporiza el agua que contienen las células diana. Se trata de un efecto específico en las capas superficiales de la piel y no causa daño en los tejidos circundantes.

Fue en el año 2003 cuando se identificó por primera vez el mecanismo principal del efecto láser, que se basa en la generación de una respuesta 
suprafisiológica en los niveles de calor que inducen una HRS local en la zona tratada. Esta respuesta origina una rápida y temporal alteración en el metabolismo celular caracterizada por un incremento masivo en la producción de las proteínas de respuesta HSP, presentes en las células del organismo. Estas proteínas juegan un papel modulador (chaperonas) en la protección del medio ambiente celular de las agresiones térmicas (87-89) y sus patrones de expresión se modifican cuando las células son expuestas a elevadas temperaturas u otros agentes $(90,91)$. Las HSP tienen un papel importante en la coordinación de la expresión de numerosos factores de crecimiento, como el TGF-beta, que es el factor clave en la respuesta inflamatoria y el proceso de neofibrogénesis. Tal y como se ha referido con anterioridad, el efecto térmico del láser activa las HSP que estimulan a los fibroblastos (células clave), que producirán colágeno maduro y nuevas fibras elásticas, incrementando la angiogénesis, el flujo vascular y la permeabilidad de la mucosa vaginal para la llegada de numerosos nutrientes a la lámina propia, formando nuevos componentes de la matriz extracelular, así como la hidratación y la regeneración del epitelio vaginal $(17,81,82)$.

El láser de CO2 microablativo y fraccionado (eliminación de las capas más superficiales de la piel con scanning fraccional de tejido mediante el sistema DOT), emite un pulso determinado en 2 fases (D-pulse): un efecto agudo termo-ablativo inicial intenso, que produce la ablación de la zona epitelial de la mucosa atrófica vaginal con bajo contenido en agua, seguido de un efecto térmico prolongado que facilita la proliferación del colágeno y del tejido conjuntivo. Gracias a esta sistemática de aplicación de la energía láser el procedimiento en la vagina es mínimamente invasivo, consiguiendo una mejoría en la hidratación y elasticidad de las paredes vaginales, aliviándose de manera importante la clínica en las mujeres menopáusica (92).

El principal objetivo del tratamiento del SGM es reducir sus síntomas. Casi el $50 \%$ de las mujeres de 60 años sanas presentan síntomas como la sequedad, el dolor y la dispareunia, que están íntimamente y consistentemente asociados con un marcado efecto negativo en las relaciones sexuales y personales, y que ocasiona una menor $\mathrm{CV}$ $(8,92)$. Distintas opciones terapéuticas han sido propuestas y como primera recomendación general se aconseja a todas las pacientes cambios en los hábitos de vida hacia un tipo más saludable (disminución del consumo de tabaco, pérdida de peso y actividad sexual regular).

La THS local y sistémica se han mostrados como las más efectivas para casos moderados y severos, y además, también disponemos de los tratamientos no hormonales vaginales (hidratantes y lubricantes) (42). Nuevos y emergentes tratamientos como el Ospemifeno (Selective Estrogen Re- ceptor Modulator-SERM) (93-99), los derivados de la Testosterona, una combinación de $\mathrm{E}$ y Bazedoxifeno (Tissue Selective Estrogen Complex-TSEC) (100-104), y el láser vaginal, han demostrado beneficios en el tratamiento de los síntomas del SGM $(7,13,14,17,38,103,104)$.

La pérdida de los niveles de $\mathrm{E}$ como consecuencia de la menopausia parece ser un punto de inflexión en la aparición de una variedad de alteraciones clínicas, incluyendo el envejecimiento cutáneo y génitourinario (103-106). Sin embargo, nuevas tendencias en los tratamientos utilizados en la menopausia $(7,26,27,42,47,94,103,104)$ incluyen numerosos tratamientos no hormonales para promover un envejecimiento saludable en todos los sentidos y, después de la publicación de los estudio Women's Health Initiative, el abordaje de todo lo relacionado con la salud urogenital y sexual ha cambiado significativamente (103-107). La THS sistémica mejora la clínica del SGM en la mayoría de las mujeres, $74 \%$ después de un año de tratamiento, pero un $26 \%$ de ellas continuarán con clínica vaginal (108). Este hecho es razón suficiente para justificar la no recomendación de iniciar la THS sistémica en mujeres con síntomas vaginales exclusivamente, y el que en muchos casos se requiera inicialmente la combinación de E sistémicos y locales, especialmente cuando se emplea esta terapia a dosis bajas (19). La terapia estrogénica local no solo reduce la mayoría de los efectos adversos de la terapia sistémica, sino que probablemente es más eficaz en el tratamiento de la sintomatología genitourinaria de la menopausia. Por ello, las mujeres que presentan SGM como única consecuencia de la deprivación estrogénica son las candidatas para recibir el tratamiento hormonal local (7-27,47,103-108).

Los tratamientos no hormonales vaginales constituyen una parte importante de la estrategia integral para promover el envejecimiento correcto de la zona urogenital, sobre todo en aquellas mujeres que no desean o no están indicadas las terapias hormonales sistémicas por diferentes motivos, como el rechazo del tratamiento por miedo, los efectos secundarios, el pobre cumplimiento, las contraindicaciones o la falta de eficacia $(53,54,109)$.

El uso de hidratantes vaginales y lubricantes no ha demostrado una eficacia ampliamente contrastada y persiste el problema de la falta de adherencia al tratamiento $(8,86,87,103,104,110,111)$. Muchas otras sustancias solas y/o en combinación, como productos derivados de plantas, ácido hialurónico, vitamina $E$ y fitoestrógenos, han sido utilizadas para tratar el SGM localmente $(30,47,103,104,109,110)$, con la intención de contrarrestar la pérdida de elasticidad e hidratación de la mucosa vaginal $(8,10,30,111)$, sin evidencia suficiente que apoye tales indicaciones $(109,111,112)$. La ausencia de resultados de estudios a largo plazo y controlados 
está bien documentada, pero lo cierto es que el impacto tan negativo que sufren las pacientes con SGM en las relaciones interpersonales, en su esfera sexual, en la pérdida de autoestima y en su calidad de vida, es recogido casi diariamente en las consultas de ginecología (31-34). Por lo tanto, los profesionales debemos ser proactivos a la hora de investigar la clínica y su repercusión local y general y ofertar a las mujeres menopáusicas las diferentes opciones terapéuticas en función de sus expectativas personales, experiencias con dichos tratamientos, preferencias y satisfacción.

Especial atención merecen las pacientes que padecen cáncer ginecológico estrógeno-dependiente (mama, ovario, endometrio), las cuales presentan un elevado riesgo de síntomas importantes de AVV y disfunción sexual, especialmente en mujeres jóvenes (113-119). La literatura ha demostrado un incremento en la incidencia y progresión de los síntomas genitourinarios consecuencia de la quimioterapia, radioterapia y de la terapia endocrina. Aproximadamente entre el $50-70 \%$ de las pacientes que han padecido un cáncer de mama presentan uno o más síntomas urogenitales. Como consecuencia de las contraindicaciones de los tratamientos clásicos, el impacto en la CV y debido a la escasa adherencia a los tratamientos locales a largo plazo, el manejo de estos efectos colaterales en este grupo de pacientes es un aspecto fundamental a tener en cuenta dentro del cuidado integral de estas mujeres. El tratamiento con E local es el más planteado actualmente pero los profesionales deben estar actualizados y basarse en las indicaciones de las guías clínicas que regulan tal recomendación. No existen estudios que permitan hacer recomendaciones de tratamiento con $E$ basadas en evidencias al respecto. Después de cualquier cáncer ginecológico puede ser apropiado un enfoque multidisciplinar en relación al uso de E y consensuar con la paciente el tratamiento. En general, para las pacientes con cáncer de mama $\mathrm{E}$ dependiente, son preferibles de inicio las terapias no hormonales, pero cuando éstas no son efectivas, pueden utilizarse $E$ vaginales en la mínima dosis eficaz y tras el consentimiento de la paciente $(16,42,116)$. Para este grupo de mujeres el tratamiento vaginal con láser de $\mathrm{CO} 2$ se plantea como una opción viable $(117,118)$.

En 2011, Gaspar y cols (69), demostraron por primera vez la mejoría histológica que aparecía en las muestras de biopsias vaginales que habían sido tratadas con el láser fraccionado microablativo de CO2 en combinación con plasma rico en plaquetas. Comprobaron los efectos beneficiosos en las tres capas de la pared vaginal en contraposición con los tratamientos con los E u otras terapias locales, que solo obtenían mejoría a nivel del epitelio $(47,93,94,119)$. Comprobaron como el sistema fraccionado del láser podía irradiar la energía a las capas más profundas de la vagina y reactivar la síntesis de colágeno y de la matriz extracelular, recuperándose el trofismo vaginal con un mínimo trauma en las zonas superficiales tratadas.

Salvatore y cols (7), en 2014, publicaron un estudio piloto durante 12 semanas de tratamiento en mujeres postmenopáusicas con AVV, utilizando el láser $\mathrm{CO} 2$ fraccionado. Sus resultados demostraron que la aplicación del láser fue efectiva y mejoró los síntomas de AVV de forma significativa (sequedad vaginal, quemazón, prurito, dispareunia y disuria; $p<0,001)$ y el VHIS $(13,1 \pm 2,5$ en la visita basal versus $23,1 \pm 1,9 ; p<0,001)$ a las 12 semanas de seguimiento. El mismo grupo demostró mejores resultados en lo relacionado con la esfera y función sexual en mujeres postmenopáusicas con AVV después de tres sesiones de láser CO2 (120). Estas publicaciones indicaban por primera vez que el laser podía ser considerado como una nueva oportunidad para el tratamiento no hormonal del SGM.

Perino y cols (17), también evaluaron la efectividad de este tratamiento en 2015 , y los resultados fueron similares a los publicados con anterioridad. Sus datos indicaban una mejoría significativa en los síntomas de AVV con una $p<0,0001$. De los resultados globales, un $91,7 \%$ de las pacientes estaban muy satisfechas o satisfechas con el procedimiento y experimentaron una considerable mejoría en su CV, sin efectos adversos debidos a la técnica. Otros grupos han publicado igualmente resultados equivalentes en este grupo de mujeres (75).

Recientemente, otro tipo de láser ha sido utilizado para aliviar la sintomatología del SGM. En 2015, Gambacciani y cols (38), publicaron un estudio piloto prospectivo y comparativo, en mujeres que padecían SGM, las cuales fueron tratadas con el láser vaginal de Erbio:YAG (VEL) (XS Fotona Smooth, Fotona, Ljubijana, Eslovenia). Se trata de un láser no ablativo, cuyo medio activo sólido es el cristal YAG (Yttrium Aluminium Garnet) dopado con Erbio (Er:YAG), frente a un grupo control tratado con una pauta conocida ultrabaja para el SGM (1 $\mathrm{g}$ de gel vaginal con $50 \mu \mathrm{g}$ de estriol 2 veces por semana), que se consideraba segura y efectiva para el tratamiento de la atrofia postmenopáusica. El tratamiento con VEL indujo un descenso significativo en la escala VAS en los síntomas de sequedad vaginal y dispareunia $(p<0,01)$, con un incremento significativo $(p<0,01)$ del WHIS, confirmando y ampliando los buenos resultados obtenidos con el VEL por Gaspar y cols (62). El VEL puede incrementar el grosor epitelial y su contenido en glucógeno, asociado a cambios en la lámina propia, aumentando la angiogénesis, la colagenosis, papilomatosis y la celularidad de la matriz extracelulara (69). Todos estos cambios fueron controlados a largo plazo y observados 6 meses después de la última sesión, persistiendo los efectos clínicos beneficiosos 24 semanas posteriores al final del tratamiento (38). 
Además, y en el contexto de manifestaciones clínicas que aparecen con frecuencia en la menopausia, se han publicado trabajos que sugieren que el tratamiento con VEL puede ser de utilidad en mujeres postmenopáusicas con síntomas de incontinencia urinaria de esfuerzo (IUE), mejorando los indicadores del cuestionario sobre calidad de vida específico (ICIQ-SF) para estas pacientes (38). Los tratamientos no quirúrgicos para la IUE constituyen un importante desafío en la actualidad y el manejo no farmacológico de la IUE, mediante los ejercicios de fortalecimiento del suelo pélvico, puede mejorar los síntomas pero desafortunadamente muchas mujeres abandonan dicha terapia. Los datos publicados respecto a la IUE indican que el tratamiento con VEL podría ser de utilidad como una alternativa no invasiva para estos casos de IUE $(38,121-123)$.

Como consecuencia de las buenas expectativas que la tecnología láser tiene como opción terapéutica no hormonal sobre el SGM y como alternativa no quirúrgica para la IUE, se ha diseñado en Italia el estudio multicéntrico VELAS (Vaginal Erbium Laser Academy Study), que se llevará a cabo en 1500 mujeres postmenopáusicas para evaluar a largo plazo (1 año) la eficacia y seguridad de la técnica VEL en estos cuadros clínicos (124).

Por otro lado, y debido a los prometedores resultados de la aplicación del láser en el canal vaginal y aunque no esté encuadrado en el ámbito de la mujer menopáusica, se ha publicado otra indicación de la técnica, basada en el mismo mecanismo de acción y consecuencia histológica del efecto térmico. Se trata de su utilidad en el Síndrome de Hiperlaxitud Vaginal (VRS), propio de mujeres en edad reproductiva y que como consecuencia de los partos sufren una pérdida de tono y elasticidad de las paredes vaginales que les ocasiona problemas físicos y psicológicos y con frecuencia en su relación de pareja.

Recientemente, el VEL, se ha mostrado relevante para los casos de VRS. Así, Min (125), en 2014, publicó un estudio piloto en 30 mujeres que habían parido y que presentaban VRS o AVV, con edades comprendidas entre 33-56 años (mediana 41,7 años). Todas las pacientes completaron satisfactoriamente el tratamiento sin objetivarse efectos adversos. Se evidenció una mejoría significativa en la relajación de la pared vaginal en todas las pacientes a los 2 meses posteriores al tratamiento basada en los valores de presión obtenidos por perineómetro, en la valoración por parte de la pareja del grado de estrechamiento vaginal $(76,6 \%)$ y por el grado de satisfacción sexual referida por las propias mujeres $(70,0 \%)$. Los hallazgos histológicos sugerían una mayor elasticidad, firmeza y estrechamiento de la vagina.

Finalmente, se está experimentando en una aplicación novedosa del láser fraccionado de CO2, mediante la administración de determinados fárma- cos conducidos por el haz de luz, cuya intención es aumentar los efectos terapéuticos de los mismos en los tejidos diana (126).

La intención de los estudios publicados hasta ahora que evidencian efectos beneficiosos en mujeres menopáusicas con SGM es, además de considerar la alternativa terapéutica en este grupo de pacientes, promover futuras investigaciones en mujeres con contraindicaciones para el tratamiento hormonal y por tanto, más difíciles de manejar (pacientes oncológicas), y en mujeres que desean asociar tratamientos hormonales y no hormonales con la tecnología láser para conseguir aliviar más la clínica del SGM. En efecto, es probable que la sinergia entre los diferentes tratamientos, aplicados conjuntamente o de forma secuencial, sea beneficiosa para las pacientes, y esto cobra mayor importancia en casos de mujeres ancianas que han sufrido durante muchos años las consecuencias de una larga deprivación estrogénica y presentan unas enormes consecuencias relacionadas con la clínica urogenital y con la estenosis vaginal (126).

Pero por otro lado, cuando el tratamiento láser se combina o continúa con una terapia local estrogénica, no podemos excluir actualmente que exista una mayor absorción del $E$ incluso en preparados de muy baja dosis, debido al incremento en la absorción de la mucosa vaginal provocada por el láser. La importancia de este efecto tisular, precisa de estudios diseñados para valorar los riesgos y beneficios del tratamiento láser en función de la historia personal de las mujeres (7).

\section{CONCLUSIÓN}

En función de los datos publicados, para el tratamiento de las mujeres con síntomas severos del SGM que no se benefician de los cambios en el estilo de vida, de los hidratantes vaginales o de la terapia con $\mathrm{E}$ locales, los ginecólogos deberíamos valorar la indicación del láser fraccionado como una opción terapéutica. Así lo recogen numerosos grupos de trabajo y el algoritmo terapéutico de la Guía Práctica de Asistencia en el Síndrome Urogenital de la Menopausia, actualizada en 2015 y auspiciada por la Sociedad Española de Ginecología y Obstetricia (13). Los resultados obtenidos de diferentes estudios piloto han demostrado que el tratamiento con láser $\mathrm{CO} 2$ microablativo fraccionado en mujeres postmenopáusicas con SGM es efectivo, fácil de realizar, seguro, bien tolerado y sin efectos adversos. Si estos hallazgos son confirmados en investigaciones futuras controladas y a largo plazo, mínimo 12 meses de seguimiento desde la última sesión, dispondremos de una novedosa alternativa segura y válida más que ofrecer a nuestras pacientes para tratar los síntomas del SGM, bien de forma aislada o asociada a terapias clásicas ya conocidas, dentro de la práctica clínica en menopausia. 


\section{REFERENCIAS}

1. Salvatore $S$, Leone U, Athanasiou $S$, et al. Histological study on the effects of microablative fractional CO2 laser on atrophic vaginal tissue: an ex vivo study. Menopause 2015;22:845-9.

2. Iosif CS, Batra S, Ek A, Astedt B. Oestrogens receptors in the human female lower urinary tract. $A m \mathrm{~J}$ Obstet Gynaecol 1981;141:817ᄀ-20.

3. Wysocki S, Kingsberg S, Krychman M. Management of vaginal atrophy: Implications from REVIVE survey. Clin Medic Insights Reprod Health 2014;8:23-30.

4. The North American Menopause Society. Management of symptomatic vulvo $\neg$ vaginal atrophy: 2013 position statement of The North American Menopause Society. Menopause 2013;20:888-902.

5. US Department of Commerce. Age and sex composition: 2010. Washington, DC: US Census Bureau. Report No.: C2010BR-03; 2011.

6. Gass ML, Cochrane BB, Larson JC, et al. Patterns and predictors of sexual activity among women in the hormone therapy trials of the Women's Health Initiative. Menopause 2011;18:1160-71.

7. Salvatore S, Nappi RE, Zerbinati N, et al. A 12-week treatment with fractional $\mathrm{CO} 2$ laser for vulvovaginal atrophy: a pilot study. Climateric 2014;17:363-9.

8. Castelo-Branco C, Cancelo MJ, Villero J, Nohales F, Juliá MD. Management of post-menopausal vaginal atrophy and atrophic vaginitis. Maturitas 2005;52 Suppl 1:S46-S52

9. Calleja-Agius J, Brincat MP. Urogenital atrophy. Climacteric 2009;12:279-85.

10. Archer DF. Efficacy and tolerability of local estrogen therapy for urogenital atrophy. Menopause 2010;17:194-203.

11. Mehta A, Bachmann G. Vulvovaginal complaints. Clin Obstet Gynecol 2008;51: 549-55.

12. Portman DJ, Gass ML. Vulvovaginal Atrophy Terminology Consensus Conference Panel. Genitourinary syndrome of menopause: new terminology for vulvovaginal atrophy from the International Society for the Study of Women's Sexual Health and The North American Menopause Society. Climateric 2014;17:557-63.

13. Palacios S, Cancelo MJ. Guía práctica de asistencia del síndrome urogenital de la menopausia (SEGO). Actualización 2015. Ed: SEGO (junio 2015). Madrid. España.

14. Hutchinson-Colas J, Segal S. Genitourinary syndome of menopause and the use of laser therapy. Maturitas 2015;82:342-5.

15. Redfern N, Gallagher P. The ageing anaesthetist. Anaesthesia 2014;69:1-5

16. MacBride MB, Rhodes DJ, Shuster LT. Vulvovaginal atrophy. Mayo Clin Proc 2010;85:87-94.

17. Perino A, Calligaro A, Forlani $F$, et al. Vulvo-vaginal atrophy: a new treatment modality using thermo-ablative fractional CO2 laser. Maturitas 2015;80:296-301.

18. Suckling J, Lethaby A, Kennedy R. Local oestrogen for vaginal atrophy in post-menopausal women. Cochrane Database Syst Rev 2003:CD001500.

19. Sturdee DW, Panay N, International Menopause Society Writing Group. Recommendations for the management of postmenopausal vaginal atrophy. Climacteric 2010;13:509-22.
20. Fredman MA. Vaginal $\mathrm{pH}$, estrogen and genital atrophy. Menopause Management 2008;17:9-13.

21. Parish SJ, Nappi RE, Krychman MI, et al. Impact of of vulvovaginal health on postmenopausal womwn: a review of survey on symptoms of vulvovaginal atrophy. Int J Women's Health 2013;5:437-47.

22. Fadare $O$. Vaginal stromal sclerosis: a distinctive stromal change associated with vaginal atrophy. Int J Gynecol Pathol 2011;30:295-300.

23. Simon JA. Identifying and treating sexual dysfunction in postmenopausal women: the role of estrogen. J Womens Health (Larchmt) 2011;20:1453-65.

24. Levine KB, W illiams RE, Hartmann KE. Vulvovaginal atrophy is strongly associated with female sexual dysfunction among sexually active postmenopausal women. Menopause 2008;5:661-6.

25. Goldstein I. Recognizing and treating urogenital atrophy in postmenopausal women. J Womens Health (Larchmt) 2010;19:425-32.

26. Rees M, Pérez-López FR, Ceasu I, et al. EMAS clinical guide: low-dose vaginal estrogens for postmenopausal vaginal atrophy. Maturitas 2012;3:171-4.

27. Archer DF. Efficacy and tolerability of local estrogen therapy for urogenital atropy. Menopause 2010;17:194-203.

28. Santoro N, Komi J. Prevalence and impact of vaginal symptoms among postmenopausal women. J Sex Med 2009;6:2133-42.

29. Pastore LM, Carter RA, Hulka BS, Wells E. Self-reported urogenital symptoms in postmenopausal women: Women's Health Initiative. Maturitas 2004;49:292303.

30. Palacios S. Managing urogenital atrophy. Maturitas 2009;63:315-18.

31. Nappi RE, Lachowsky M. Menopause and sexuality: prevalence of symptoms and impact on quality of life. Maturitas 2009;63:138-41.

32. Nappi RE, Palacios S. Impact of vulvovaginal atrophy on sexual health and quality of life at postmenopause. Climacteric 2014;17:3-9.

33. Nappi RE, Kokot-Kierepa M. Women's voices in the menopause: results from an international survey on vaginal atrophy. Maturitas 2010;67:233-8.

34. Nappi RE, Kokot-Kierepa M. Vaginal Health: Insights, Views and Attitudes (VIVA) - results from an international survey. Climacteric 2012;15:36-44.

35. Ibe C, Simon JA. Vulvovaginal atrophy: current and future therapies (CME). J Sex Med 2010;7:1042-50.

36. Nappi RE, Kingsberg S, Maamari R, Simon J. The CLOSER (Clarifying Vaginal Atrophy's Impact On Sex and Relationships) Survey: Implications of vaginal discomfort in postmenopausal women and in male partners. J Sex Med 2013;10:2232-41.

37. Leiblum S, Bachman G, Kemmann E, Colburn D, Swartzman L. Vaginal atrophy inthe postmenopausal woman. The importance of sexual activity and hormones. JAMA 1983;249:2195-8.

38. Gambacciani M, Levancini M, Cervigni M. Vaginal erbium laser: the second-generation thermotherapy for the genitourinary syndrome of menopause. Climateric 2015;18:1-7.

39. Lester J, Pahouja G, Andersen B, Lustberg M. Atropic vaginitis in breast cancer survivors: a difficult survivorship issue. J Pers Med 2015;5:50-66. 
40. Kyvernitakis I, Ziller V, Hars O, Bauer M, Kalder M, Hadji P. Prevalence of menopausal symptoms and their influence on adherence in women with breast cancer. Climateric 2011;17:252-9.

41. The 2012 hormone therapy position statement of the North American Menopause Society. Menopause 2012;19:257-71.

42. de Villers TJ, Pines A, Panay $\mathrm{N}$ et al., on behalf of the International Menopause Society. Update 2013. International Menopause recommendations on menopausal hormone therapy and preventive strategies for midlife health. Climateric 2013;16:316-37.

43. Cano A, Estévez J, Usandizaga R, et al. The therapeutic effect of a new ultra low concentration estriol gel formulation $(0.005 \%$ estriol Vaginal gel) on symptoms and signs of postmenopausal vaginal atrophy: results from a pivotal phase III study. Menopause 2012;19:1130-9.

44. Weisberg E, Ayton R, Darling G, et al. Endometrial and vaginal effects of low-dose estradiol delivered by vaginal ring or vaginal tablet. Climateric 2005;8:83-92.

45. Castelo-Branco C, Hernández C. Atrofia genitourinaria. Ed: Mayo SA. , 2015.

46. Oge T, Hassa H, Aydin Y, Yalcin OT, Colak E. The relationship between urogenital symptoms and climateric complaints. Climateric 2013;16:646-52.

47. Baquedano L, Beltrán $\mathrm{E}$, Durán $\mathrm{M}$, et al. Menoguía Salud Vaginal (AEEM). Editor: Manubens M. 2014. Ed: Aureagrafic. Barcelona.

48. Portman DJ, Bachmann GA, Simon JA; Ospemifene Study Group. Ospemifene, a novel selective estrogen receptor modulator for treating dyspareunia associated with postmenopausal vulvar and vaginal atrophy. Menopause 2013;20:623-30.

49. Goldstein SR, Bachmann GA, Koninckx PR, Lin VH, Portman DJ, Ylikorkala O. The Ospemifene Study Group. Ospemifene 12-month safety and efficacy in postmenopausal women with vulvar and vaginal atrophy. Climacteric 2014;17:173-82.

50. Johnston SL, Farrell SA, Bouchard C, Farrell SA, Beckerson LA, Comeau M, et al. The detection and management of vaginal atrophy. J Obstet Gynaecol Can 2004;26:503-5.

51. Shulman LP, Portman DJ, Lee WC, et al. A retrospective managed care claims data analysis of medication adherence to vaginal estrogen therapy: implications or clinical practice. J Womens Health (Larchmt) 2008;17:569-578.

52. Kingsberg SA, Wysocki S, Magnus L, Krychman ML. Vulvar and vaginal atrophy in postmenopausal women: findings from the REVIVE (REal Women's Vlews of Treatment Options for Menopausal Vaginal Changes) survey. J Sex Med 2013;10:1709-19.

53. Kingsberg SA, Krychman ML. Resistance and barriers to local estrogen therapy in women with atrophic vaginitis. J Sex Med 2013;10:1567-74.

54. Ronconi L, Galli M. MonaLisa Touch TM. The latest frontier in the treatment of vaginal atrophy. Scientific Series, September, vol. 2. DEKA ed.; 2012.

55. Sadovsky R, Basson R, Krychman M, et al. Cancer and sexual problems. J Sex Med 2010;7:349-73.

56. Pruthi S, Simon JA, Early AP. Current overview of the management of urogenital atrophy in women with breast cancer. Breast J 2011;17:403-8.
57. Dew JE, Wren BG, Eden JA. A cohort study of topical vaginal estrogen therapy in women previously treated for breast cancer. Climateric 2003;6:45-52.

58. Biglia N, Peano E, Sgandurra P, et al. Low-dose vaginal estrogens or vaginal moisturirer in breast cancer survivors with urogenital atrophy: a preliminary study. Ginecol Endocrinol 2010;26:404-12.

59. Ong MW, Bashir SJ. Fractional laser resurfacing for acne scars: a review. Br J Dermatol 2012;166:1160-9.

60. Tierney EP, Hanke CW. Fractionated carbon dioxide laser treatment of photoaging: prospective study in 45 patients and review of the literature. Dermatol Surg 2011;37:1279-90.

61. Tierney EP, Hanke CW. Ablative fractionated CO2, laser resurfacing for the neck: prospective study and review of the literature. J Drugs Dermatol 2009;8:72331.

62. Peterson JD, Goldman MP. Rejuvenation of the aging chest: a review and our experience. Dermatol Surg 2011;37:555-71.

63. US Food and Drug Administration letter to Paolo Peruzzi. September 5, 2014. www.accessdata.fda.gov/ cdrh.docs/pdf13/K133895.pdf (accessed 23.07.2015).

64. Kaufmann R, Hibst R. Pulsed Erbium: YAG laser ablation in cutaneous surgery. Lasers Surg Med 1996;19:324-30.

65. Reynolds N, Cawrse N, Burge T, Kenealy J. Debridement of a mixed partial and full thickness burn with an erbium: YAG laser. Burns 2003;29:183-88.

66. Baraldi CE, Puricelli E, Kulkes S, Martins GL. Er: YAG laser in oral soft tissue surgery. J Oral Laser Applications 2001;1:24.

67. Levy JL, Trelles MA. New operative technique for treatment of xanthelasma palpebrarum: laser inverted resurfacing: preliminary report. Ann Plast Surg 2003;50:339-43.

68. Vizintin Z, Rivera M, Fistonic I, et al. Novel minimally invasive VSP Er: YAG laser treatments in gynecology. $\mathrm{J}$ Laser Health Acad 2012;1:46-58.

69. Gaspar A. Comparison of new minimally invasive Er: YAG laser treatment and hormonal replacement therapy in the treatment of vaginal atrophy. Climacteric 2014;17(Suppl 1):124.

70. Sasaki GH, Travis HM, Tucker B. Fractional CO2 laser resurfacing of photoaged facial and non facial skin: histologic and clinical results and side effects. J Cosmet Laser Ther 2009;11:190-201.

71. Weiss ET, Chapas A, Brightman L, et al. Successful treatment of atrophic postoperative and traumatic scarring with carbon dioxide ablative fractional resurfacing. Arch Dermatol 2010;146:133-40.

72. Berlin AL, Hussain M, Phelps R, Goldberg DJ. A prospective study of fractional scanned nonsequential carbon dioxide laser resurfacing: a clinical and histopathologic evaluation. Dermatol Surg 2009;35:222-8.

73. Lee CJ, Park JH, Ciesielski TE, Thomson JG, Persing JA. Retinoids, 585-nm laser, and carbon dioxide laser: a numeric comparison of neocollagen formation in photoaged hairless mouse skin. Aesthetic Plast Surg 2008;32:894-901.

74. Dafforn TR, Della M, Miller AD. The molecular interactions of heat shock protein 47 (Hsp47) and their implications for collagen biosynthesis. J Biol Chem 2001;276:49310-9. 
75. Zerbinati N, Serati M, Origoni M, et al. Microscopic and ultraestructural modification of postmenopausal atrophic vaginal mucosa after fractional caron diosxido laser treatment. Laser med Sci 2015;30:429-36.

76. Capon A, Mordon S. Can thermal lasers promote skin wound healing? Am J Clin Dermatol 2003;4:1-12.

77. Prignano $F$, Campolmi $P$, Bonan $P$, et al. Fractional CO2 laser: a novel therapeutic device upon photobiomodulation of tissue remodelling and cytokine pathway of tissue repair. Dermatol Ther 2009;22(suppl 1):S8-S15.

78. Ettinger B, Hait $H$, Reape KZ, Shu H. Measuring symptom relief in studies of vaginal and vulvar atrophy: the most bothersome symptom approach. Menopause 2008;15:885-9.

79. Bump RC, Mattiasson A, Bo K, et al. The standardization of terminology of female pelvic organ prolapse and pelvic floor dysfunction. Am J Obstet Gynecol 1996;175:10-17.

80. Bachmann GA, Notelovitz M, Kelly SJ, et al. Longterm nonhormonal treatment of vaginal dryness. Clin Pract Sexuality 1992;8:3-8.

81. Brizzolara S, Killeen J, Severino R. Vaginal pH and parabasal cells in postmenopausal women. Obstet Gynecol 1999;94:700-3.

82. Adelman MR, Tsai LJ; Tangchitnob EP, Kahn BS. Laser technology and application in gynaecology. J Obstet Gynaecol 2013;33:225-31.

83. Patel CKN. Selective excitayion through vibrational energy transfer and optical maser action in N2-CO2. Physical Review Letters 1964;13:617-9.

84. Bellina JH. Carbon disoxide lasers in gynecology. Obstet Gynecol Annual 1977;6:371-91.

85. Bellina $\mathrm{JH}$. Microsurgery of the fallopian tube with the carbon dioxide laser: Analysis of 230 cases with a two year follow-up. Lasers Surg Med 1983;3:255-60.

86. Townsend DE, Levin RU, Crun CP, Richart RM. Treatment of vaginal carcinoma in situ with carbon dioxide laser. Am J Obstet Gynecol 1982;143:565-8.

87. De Maio A. Extracellular heat shock proteins, cellular export vesicles, and theStress Observation System: a form of communication during injury, infec-tion, and cell damage. It is never known how far a controversial findingwill go! Dedicated to Ferruccio Ritossa. Cell Stress Chaperones 2011;16:235-49.

88. Snoeckx LHEH, Cornelussen RN, Van Neiuwenhoven FA, Reneman RS, VanderVusse GJ. Heat shock proteins and cardiovascular pathophysiology. Physiol Rev 2002;81:1461-97.

89. Yamasaki A, Tamamura $K$, Sakurai $Y$, Okuyama $N$, Yusa J, Ito $\mathrm{H}$. Remodelin-gof the rat gingiva induced by $\mathrm{CO} 2$ laser coagulation mode. Lasers Surg Med 2008;40:695-703.

90. Chen WR, Lu H, Nordquist RE. Mechanism of laser immunotherapy - role ofimmunoadjuvant and selective photothermal laser-tissue interaction. Proc SPIE 2002;4536:82-9.

91. Wang S, Diller KR, Aggarwal SJ. Kinetics study of endogenous heat shock protein 70 expression. J Biomech Eng 2003;125:794-7.

92. Roncolli L, Galli M. MonaLisa Touch: the latest frontier in the treatment of vaginal athropy. Scientific Series. DEKA ed: May 2015 (Italy).

93. Gaspar A, Addamo G, Brandi H. Vaginal fractional $\mathrm{CO} 2$ laser: a minimally invasive option for vaginal rejuvenation. Am J Cosmet Surg 2011;28:156-62.
94. Palacios S, Cancelo MJ, Castelo-Branco C, González S, Olaya MA. Recomendaciones de la Sociedad Española de Ginecología y Obstetricia sobre la Prevención y Tratamiento de la Atrofia Vaginal. Prog Obst Gynecol 2012;55:408-15.

95. McCall JL, De Gregorio MW. Pharmacologic evaluation of ospemifenene. Expert Opin Drug Metab Toxicol 2010;6:773-9.

96. Bachmann G. Ospemifene effectively treats vulvovaginal atrophy in postmenopausal women: results from a pivotal phase 3 study. Menopause 2010;17:480-6.

97. Simon JA, Lin VH, Rodovich C, Bachmann GA. The Ospemifene Study Group. One year long-term safety extension study of ospemifene for the treatment of vulvar and vaginal atrophy in postmenopausal women with a uterus. Menopause 2013;20:418-27.

98. Portman DJ, Bachmann GA, Simon JA. Ospemifene Study Group. Ospemifene, a novel selective estrogen receotor modulator for treating dyspareunia associated with postmenopausal vulvar and vaginal atrophy. Menopause 2013;20:623-30.

99. Portman D, Palacios S, Nappi RE, Mueck AO. Ospemifene, a non-oestrogen selective receptor modulator for the treatment of vaginal dryness associated with postmenopausal vulvar and vaginal atrophy: a randomized, placebo-controlled, phase III trial. Maturitas 2014;78:91-8.

100.Lobo RA, Pinkerton JV, Gass ML, et al. Evaluation of bazedoxifene/conjugated estrogens for the treatment of menopausal symptoms and effects on metabolic parameters and overall safety profile. Fertil Steri 2009;92:1025-38.

101.Pinkerton JV, Utian WH, Constantine GD, et al. Relief of vasomotor symptoms with the tissue-selective-estrogen complex containing bazedoxifene/conjugated estrogens: a ransomized controlled trial. Menopause 2009;16:1116-24.

102.Kagan R, Williams RS, Pan K, Murkin S, Pickar JH. A randomized placebo-and active-controlled trial of bazedoxifene/conjugated estrogens for treatment of moderate to severe vulvar/vaginal atrophy in postmenopausal women. Menopause 2010;17:281-9.

103.Palacios S, Mejía A, Neyro JL. Treatment of the genitourinary syndrome of menopause. Climateric 2015;18:sup1:23-9.

104.Palacios S, Castelo-Branco C, Currie H, et al. Update on management of genitourinary syndrome of menopause: A practical guide. Maturitas 2015;82:308-13.

105.Brincat MP, Baron YM, Galea R. Estrogens and the skin. Climacteric 2005;8:110-23.

106.Sartori MG, Feldner PC, Jarmy-Di Bella ZI, et al. Sexual steroids in urogynecology. Climacteric 2011;14:5-14.

107.Nappi RE, Davis SR. The use of hormone therapy for the maintenance of urogynecological and sexual health post WHI. Climacteric 2012;15:267-74.

108.Barnabei VM, Cochrane BB, Aragaki AK, et al. Menopausal symptoms and Q6 treatment-related effects of estrogens and progestin in the Women's Health Initiative. Obstet Gynecol 2005;105:1063-73.

109.Sinha A, Ewies AA. Non-hormonal topical treatment of vulvovaginal atrophy: an up-to-date overview. Climacteric 2013;16:305-12.

110.Nachtigall E. Comparative study: Replens versus local estrogen in menopausal women. Fertil Steril 1994;61:178-80. 
111.Lima SM, Bernardo SF, Yamada SS, Reis BF, da Silva GM, galvao MA. Effects of Glycine max L merr. Soy isoflavonee vaginal gel on epithelium morphology and estrogen receptor expression in postmenopausal women: a 12 week, randomized, double-blind, placebocontrolled trial. Maturitas 2014;78:205-11.

112. Hidalfo LA, Chedraui PA, Morocho N, Ross S, san Miguel G. The effect of red clover isoflavones on menopausal symptoms, lipids and vaginal cytology in menopausal women: a randomized, double blind, placebo-controlled study. Gynecol Endocrinol 2005;21:257-64.

113.Sadovsky R, Basson R, Krychman M, Morales AM, Schover L, Wang R, et al. Cancer and sexual problems. J Sex Med 2010;7:349-73.

114.Chin SN, Trinkaus M, Simmons C, et al. Prevalence and severity of urogenital symptoms in postmenopausal women receiving endocrine therapy for breast cancer. Clin Breast Cancer 2009;9:108-17.

115.Biglia N, Moggio G, Peano E, et al. Effects of surgical and adjuvant therapies for breast cancer on sexuality, cognitive functions, and body weight. J Sex Med 2010;7:1891-900.

116.Pfeiler GP, Glatz C, Königsberg R, Geisendorfer T, Fink-Retter A, Kubista E, et al. Vaginal estriol to overcome side-effects of aromatase inhibitors in breast cancer patients. Climacteric 2011;14:339-44.

117.Eden J. Endocrine dilemma: Managing menopause after breast cancer. Eur J Endocrinol 2016;174:R71-7.

118.Biglia N, Bounous VE, Sgro LG, DÁlonzo M, Pecchio $\mathrm{S}$, Nappi RE. Genitourinary syndrome of menopause in breast cancer survivors: Are we facing new and safe hopes? Clin Breast Cancer 2015;15:413-20.

119. Tierney EP, Hanke CW. Fractionated carbon dioxide laser treatment of photoaging: prospective study in 45 patients and review of the literature. Dermatol Surg 2011;37:1279-90.

120.Salvatore S, Nappi RE, Parma M et al.: Sexual function after fractional microablative $\mathrm{CO} 2$ laser in women with vulvovaginal atrophy. Climateric 2015;18:219-25.

121. Fistonic I. Laser treatment of early stages of stress urinary incontinence significantly improves sexual life. Presented at Annual Conference of European Society for Sexual Medicine, December 2012, Amsterdam.

122.Fistonic I. Erbium laser treatment for early stages of stress urinary incontinence (SUI) in women. Presented at Annual Meeting of International Urogynecological Association, May 2013, Dublin.

123.Fistonic I. Erbium laser treatment for early stages of stress urinary incontinence (SUI) and vaginal relaxation signifi cantly improves pelvic floor function. Presented at 15th Congress of Human Reproduction, March 2013, Venice.

124.Gambacciani M, Torelli MG, Martella L, et al. Rationale and design for the Vaginal Erbium Laser Academy Study (VELAS): an international multicenter study on genitourinary síndrome of menopause and stress urinary incontinence. Climateric 2015;18(Suppl 1):43-8.

125. Lee MS. Treatment of vaginal relaxation syndrome with an Erbium:YAG laser using $90^{\circ}$ and $360^{\circ}$ scanning scopes: a pilot study and short- term results. Laser Ther 2014;23:129-38.

126. Oni G, Brown SA, Kenkel JM. Can fractional lasers enhance transdermal absorption of topical lidocaine in an in vivo animal model. Lasers Surg Med 2012;44:168-74. 